\title{
ACEPTABILIDAD DE UNA INTERVENCIÓN BASADA EN SALUD MÓVIL PARA MODIFICAR ESTILOS DE VIDA EN PREHIPERTENSOS DE ARGENTINA, GUATEMALA Y PERÚ: UN ESTUDIO PILOTO
}

\author{
Andrea Beratarrechea ${ }^{1, a}$, Francisco Diez-Canseco ${ }^{1, b}$, Ariel Fernández ${ }^{1, c}$, Rebeca Kanter ${ }^{1, d}$, \\ Paola Letona ${ }^{3, b}$, Homero Martinez ${ }^{4,5, d}$, J. Jaime Miranda ${ }^{2, a, d}$, Manuel Ramirez-Zea ${ }^{3, d}$, Adolfo Rubinstein ${ }^{1, a, d}$
}

\begin{abstract}
RESUMEN
Objetivos. Evaluar la aceptabilidad y factibilidad de una intervención basada en salud móvil, para la adopción de estilos de vida saludables en personas prehipertensas que viven en zonas urbanas de bajos recursos en Argentina, Guatemala y Perú. Materiales y métodos. Se reclutaron prehipertensos entre 30 a 60 años para un estudio piloto. La intervención incluyó dos llamadas de consejería realizadas por una nutricionista, seguidas de un mensaje de texto customizado semanal. Una plataforma basada en Internet ofreció el soporte para la implementación de la intervención. Utilizando entrevistas semiestructuradas se evaluó el alcance y la aceptabilidad de esta intervención en los participantes, y la facilidad de uso en las nutricionistas. Resultados. Se logró contactar a 43 de los 45 participantes (95\%). El número promedio de llamadas para contactar a un sujeto fue de dos, con un rango de 1-9 llamadas. Dos participantes pudieron ser contactados en su teléfono celular y cinco no recibieron una exposición completa a la intervención. Basados en las entrevistas semiestructuradas, los resultados mostraron una buena aceptabilidad a la intervención en los participantes. Las nutricionistas percibieron a la plataforma como amigable y de fácil manejo. Las barreras para ofrecer esta intervención se relacionaron con dificultades para obtener una señal de telefonía celular adecuada. Conclusiones. Dada la alta penetración de la telefonía celular en países en desarrollo, se concluye que una intervención basada en salud móvil es factible y aceptable para ofrecer una intervención orientada a la modificación del estilo de vida en prehipertensos o personas de alto riesgo de enfermedades crónicas.
\end{abstract}

Palabras clave: Prehipertensión; Evaluación de tecnologías de salud; Informática médica (fuente: DeCS BIREME).

\section{ACCEPTABILITY OF A MOBILE HEALTH BASED INTERVENTION TO MODIFY LIFESTYLES IN PREHYPERTENSIVE PATIENTS IN ARGENTINA, GUATEMALA AND PERU: A PILOT STUDY}

\begin{abstract}
Objectives. To evaluate the acceptability and feasibility of an intervention based on mobile health, for the adoption of healthy lifestyles in prehypertensive people living in low-income urban areas in Argentina, Guatemala and Peru. Materials and methods. Prehypertensive people aged 30-60 years were recruited for a pilot study. The intervention included two counseling calls made by a nutritionist followed by a weekly customized text message. An internet-based platform offered support for the implementation of the intervention. Using semi-structured interviews, we evaluated the reach and acceptability of the intervention in the participants and ease of use for the nutritionists. Results. It was possible to contact 43 of the 45 participants $(95 \%)$. The average number of calls to contact a subject was two, with a range of 1-9 calls. Two participants could not be reached on their cell phone; five did not receive complete exposure to the intervention. Based on semi-structured interviews, the results showed good acceptability for the intervention by the participants. Nutritionists perceived the platform as friendly and easy to use. Barriers to deliver this intervention were related to difficulties in obtaining an adequate cellular signal. Conclusions. Given the high penetration of mobile phones in developing countries, it is concluded that it is feasible and acceptable to offer a mobile health based intervention oriented towards lifestyle modification in people with prehypertension or high risk of chronic disease intervention.
\end{abstract}

Key words: Prehypertension; Technology assessment, biomedical; Medical informatics (source: MeSH NLM).

\footnotetext{
Centro de Excelencia en Salud Cardiovascular para el Cono Sur (CESCAS). Instituto de Efectividad Clinica y Sanitaria (IECS). Argentina.

CRONICAS Centro de Excelencia en Enfermedades Crónicas, Universidad Peruana Cayetano Heredia. Lima, Perú.

INCAP Centro de Investigación Integral del INCAP para la Prevención de las Enfermedades Crónicas, Instituto de Nutrición de Centro América y Panamá. Guatemala.

Corporacion RAND. EE.UU.

Hospital Infantil de Mexico "Dr. Federico Gómez". México

Médico, ${ }^{\mathrm{b}} \mathrm{MPH},{ }^{\mathrm{c}} \mathrm{MCs},{ }^{\mathrm{d}} \mathrm{PhD}$.

Recibido: : 10-03-15 Aprobado: 13-05-15
}

Citar como: Beratarrechea A, Diez-Canseco F, Fernández A, Kanter R, Letona P, Martinez H, et al. Aceptabilidad de una intervención basada en salud móvil para modificar estilos de vida en prehipertensos de Argentina, Guatemala y Perú: un estudio piloto. Rev Peru Med Exp Salud Publica. 2015;32(2):221-9. 


\section{INTRODUCCIÓN}

Las enfermedades cardiovasculares (ECV) representan una carga pesada sobre los sistemas de atención a la salud, y afectan la economía y el bienestar de individuos, familias y a la comunidad en general, especialmente en los países de bajos y medianos ingresos ${ }^{(1,2)}$. Existe una fuerte relación lineal entre los niveles de presión arterial elevada y el riesgo de ECV ${ }^{(3)}$. De hecho, la prevalencia de hipertensión arterial (HTA) y de prehipertensión se encuentra entre el $25-34 \%$ en la población de Argentina, Guatemala y Perú (4-6).

Las intervenciones tempranas para aumentar la práctica de estilos de vida saludables en individuos con alto riesgo de desarrollar hipertensión pueden reducir la presión arterial (PA) y su tasa de progresión hacia la HTA, e inclusive prevenir completamente su desarrollo ${ }^{(7-9)}$. Sin embargo, los sistemas de atención primaria a la salud en los países en desarrollo carecen de programas efectivos orientados a la modificación de estilos de vida.

La promoción de la salud está mostrando un cambio hacia nuevas modalidades de ofrecer atención preventiva, apoyándose en tecnologías de la información y las comunicaciones para alcanzar a una mayor proporción de la población. Sin embargo, la evidencia disponible sobre la efectividad de las intervenciones basadas en e-Salud para modificar estilos de vida aún es escasa y poco clara. Los efectos reportados son pequeños, variables, y el alcance se encuentra limitado a mujeres con altos niveles de educación en países con altos ingresos ${ }^{(10,11)}$.

La salud móvil se refiere al uso de la telecomunicación móvil y tecnologías multimedia para ofrecer atención a la salud ${ }^{(12,13)}$. Con el rápido crecimiento que ha tenido el uso de la telefonía celular en países de bajos y medianos ingresos, esta tecnología está surgiendo como una herramienta útil para enfrentar muchas de las limitantes que presentan los sistemas de atención a la salud en países en vías de desarrollo, tales como la existencia de una fuerza de trabajo limitada y con excesiva carga de trabajo; restricción en los recursos económicos para la atención, así como el aumento reciente en la prevalencia de las enfermedades crónicas. Atender a los problemas de salud pública con todas estas limitantes resulta difícil, especialmente en países de bajos y medianos ingresos que buscan extender la cobertura a poblaciones de difícil acceso ${ }^{(14)}$.

Las estrategias de salud móvil, recurriendo ya sea al uso de llamadas por teléfono celular o a mensajes de texto (SMS por sus siglas en inglés: short message system) han mostrado que pueden mejorar la comunicación entre el proveedor de salud y el paciente, motivar el cambio en el comportamiento, y ayudar al manejo de algunas enfermedades crónicas ${ }^{(15-18)}$. Asimismo, la salud móvil ha mostrado resultados positivos para la promoción de la pérdida de peso, el aumento en la actividad física, y en la cesación tabáquica ${ }^{(15,19)}$. Sin embargo, el desarrollo de la salud móvil, por sí sola, no es suficiente para mejorar los resultados en la salud pública en países de bajos y medianos ingresos. El impacto en la salud poblacional solo podrá lograrse si las nuevas tecnologías se integran al sistema de salud y logran una amplia aceptación y uso.

En este trabajo se presentan los resultados de un estudio piloto que se llevó a cabo para evaluar la aceptabilidad de una intervención basada en salud móvil orientada a la modificación de estilos de vida en individuos con prehipertensión que viven en medios urbanos de bajos recursos en Argentina, Guatemala y Perú. De igual forma, se describe el desarrollo de una plataforma basada en Internet para apoyar la intervención de salud móvil.

\section{MATERIALES Y MÉTODOS}

\section{DISEÑO DEL ESTUDIO Y RECLUTAMIENTO}

Se realizó un estudio piloto, de tres meses de duración, con el objeto de probar la factibilidad y aceptabilidad a la intervención basada en salud móvil. El método de muestreo fue no probabilístico, por conveniencia y se reclutaron 45 individuos para tomar parte en el estudio. El reclutamiento de los participantes se llevó a cabo en centros de salud localizados en zonas urbanas de bajos recursos en Argentina (Bajo Boulogne y Mar del Plata, Provincia de Buenos Aires), y en Guatemala (Villa Nueva) y en el Hospital Cayetano Heredia en Lima, Perú; aprovechando la visita de estos individuos al centro de salud durante el mes de noviembre de 2011. Los individuos elegibles para el estudio fueron hombres y mujeres entre 30 a 60 años, con prehipertensión (presión arterial sistólica entre 120-139 o presión diastólica entre $80-90 \mathrm{~mm} \mathrm{Hg}$ ), que no estuvieran recibiendo medicación para controlar la presión arterial, y que contaran con un teléfono celular para su uso personal. Los criterios de exclusión fueron la presencia de embarazo, diabetes mellitus, HTA, antecedentes de infarto al miocardio o accidente cerebrovascular y ser analfabeto.

En los centros de salud, médicos o enfermeras llevaron a cabo la práctica de antropometría y toma de presión arterial, así como la administración de un cuestionario a los individuos que aceptaron participar. El cuestionario recolectó información sobre características demográficas y socioeconómicas; utilización de servicios de salud; hábitos dietarios; actividad física; y patrones de uso del teléfono celular. Los datos recolectados fueron ingresados a una 
plataforma de administración de datos basada en Internet, y se generó un registro para cada participante que contenía información personal y clínica, incluyendo nombre, sexo, edad, número de teléfono celular, índice de masa corporal, presión arterial, y hábitos dietéticos. Esta información fue utilizada para personalizar la intervención ofrecida.

\section{DESCRIPCIÓN DE LA INTERVENCIÓN DE SALUD MÓVIL}

En cada país se ofreció un entrenamiento de tres días de duración a dos nutricionistas; se las capacitó en cómo ofrecer la consejería requerida a través de una llamada por teléfono celular, basándose en la técnica de entrevista motivacional ${ }^{(20)}$. La capacitación fue realizada por una de las investigadoras del proyecto, especialista en cambio de comportamientos y psicología en salud, y consistió en una capacitación introductoria sobre la entrevista motivacional, esta incluyó contenidos teóricos y talleres prácticos.

De igual forma, se las capacitó sobre cómo usar la plataforma basada en Internet para apoyar la entrega de las intervenciones propuestas. A lo largo de un mes, los participantes incluidos recibieron una llamada corta de introducción, en la cual la nutricionista presentó el estudio; a esta le siguió una segunda llamada en la cual el participante recibió la consejería de la nutricionista sobre uno de las siguientes comportamientos específicos: reducción de la ingestión de sodio; reducción en la ingestión de azúcares simples y grasas saturadas; aumento en el consumo de frutas y verduras, y promoción de la actividad física. Las nutricionistas llevaron a cabo una evaluación de la etapa de cambio en que se encontraba cada sujeto para cada una de las conductas identificadas para el cambio, basándose en el modelo transteórico, el cual describe las etapas de precontemplación, contemplación, preparación, acción y mantenimiento. Se ofrecieron consejerías siguiendo el protocolo de la entrevista motivacional (20-22). Con esta evaluación se orientó la consejería de forma individual para motivar a cada participante a realizar cambios en su estilo de vida relacionados con la conducta específica que se buscaba modificar. La información obtenida durante la llamada de consejería relativa a la disposición del sujeto para llevar a cabo modificaciones en su estilo de vida fue ingresada por la nutricionista a la plataforma informática.

Posterior a esta llamada, cada participante recibió semanalmente un mensaje de texto con información relacionada a la conducta identificada durante la llamada. Para cada una de estas conductas se desarrollaron y validaron los mensajes específicos para cada etapa de cambio. Todos los SMS fueron validados y adaptados culturalmente en cada país ${ }^{(23)}$. Con base en los datos de los participantes, la plataforma utilizó algoritmos específicos para procesar la información y generar un grupo de mensajes individualizados que se enviaron a cada participante a lo largo del mes.

\section{LA PLATAFORMA BASADA EN INTERNET}

Se construyó una plataforma basada en la web a fin de facilitar la entrega de la intervención de salud móvil. Esta plataforma permite adaptaciones para los usuarios (nutricionistas), y también permite adaptar las necesidades de los investigadores para acceder y analizar la información capturada. Para asegurar la confidencialidad de la información se utilizó un servidor exclusivo para el proyecto, y se siguió el protocolo de capa de conexión segura o Secure Sockets Layer (SSL), así como un código personal de acceso (nombre de usuario) y contraseña para las nutricionistas y los investigadores. Esta plataforma opera en el sistema Linux, está apoyada en aplicaciones de Apache Web Server, Java, MySQL y Tomcat; la encriptación de los datos se autoriza mediante certificación de SSL. Los datos pueden ser importados y exportados desde la plataforma mediante archivos en formato MS Excel (Microsoft Corporation, Redmond, WA, USA), el cual también permite el análisis estadístico.

La plataforma incluye: 1) información basal del participante, a fin de que la nutricionista la tenga accesible durante la llamada de consejería; 2) una agenda para programar las llamadas mensuales con los participantes; 3) una base de datos para recolectar información sobre la llamada en relación con el comportamiento identificado para el cambio, así como la etapa de cambio en la que se encontraba cada participante; 4) un sistema automatizado que mapea una taxonomía de etapas de cambio y conductas específicas, a fin de individualizar el mensaje de texto que sería enviado a cada participante. La plataforma utiliza algoritmos específicos para procesar los datos y recuperar un grupo de SMS personalizados que se envían a cada participante a lo largo de un mes, y 5) informes de avance para monitorear el proceso de la entrega de la intervención, en cada país (por ejemplo, el número de SMS enviados a cada participante, o el número de llamadas de consejería logradas) (Figura 1 y Figura 2).

Se utilizó como herramienta el software de acceso libre FrontlineSMS (FrontlineSMS software program) para que las nutricionistas pudieran enviar los mensajes semanales de texto generados por la plataforma ${ }^{(24,25)}$.

\section{MEDIDAS DE RESULTADO}

La información de las llamadas de consejería y de los mensajes de texto recolectadas en la plataforma se utilizó para evaluar cómo se llevó a cabo la intervención. Se evaluó el alcance sobre la base de la proporción de 


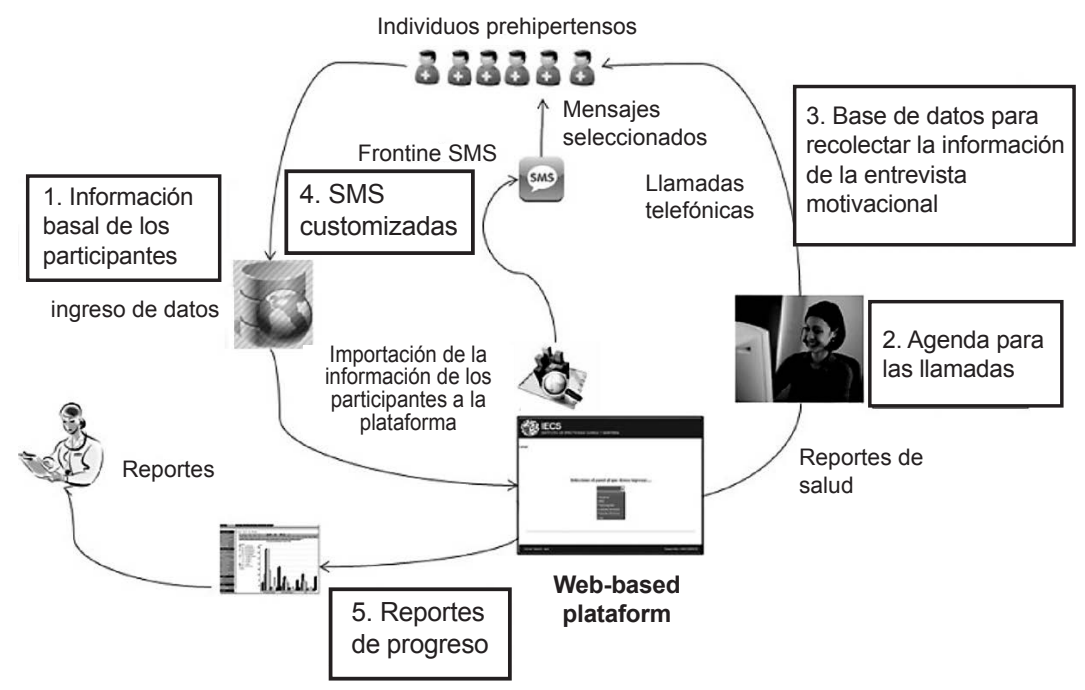

Figura 1. Componentes de la plataforma basada en Internet

la población objetivo que recibió la intervención. A fin de evaluar qué tan bien se implementó la intervención se determinó el porcentaje de participantes que completó la intervención. Las pérdidas fueron definidas como el porcentaje de participantes que abandonaron la intervención.

\section{MÉTODOS CUALITATIVOS}

Se llevó a cabo entrevistas a profundidad con los participantes para determinar la aceptabilidad a la intervención, asimismo, entrevistas con las nutricionistas a fin de evaluar si la plataforma diseñada para apoyar la intervención fue útil y si se encontraban satisfechas con el desempeño de la herramienta. Tres de los autores de este trabajo llevaron a cabo las entrevistas a lo largo de un mes, utilizando una guía semiestructurada previamente revisada y consensada entre ellos.
El protocolo de entrevista evaluó la percepción de los participantes en cuanto al contexto, estilo y lenguaje utilizado durante la llamada, así como sobre los mensajes de texto recibidos. Además, se exploró si la intervención fue aceptable, si se entendió, y si fue útil para promover la modificación en los estilos de vida; se buscó identificar qué problemas surgieron durante la llamada y durante la recepción de los mensajes de texto. Las entrevistas se grabaron en formato digital para su posterior transcripción y resumen, a partir de donde se eligieron las citas textuales incluidas en los resultados.

El análisis de la información se basó en el análisis crítico del discurso. Se exploró la función textual, donde se capta lo que los participantes dicen, los temas emergentes y su magnitud; la función relacional ocupada de las interacciones y los términos en que se dan, y la función ideacional que explora las creencias e ideas de los participantes.

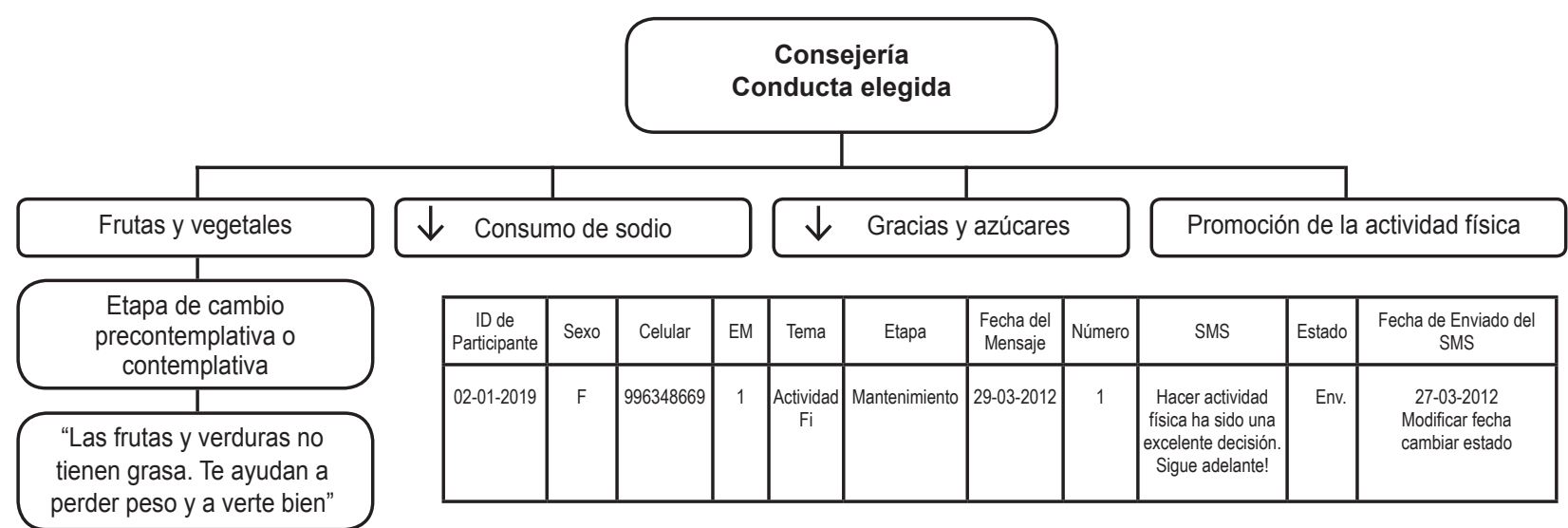

Figura 2. Algoritmo para individualizar los mensajes de texto 


\section{ANÁLISIS ESTADÍSTICO}

Los análisis estadísticos se hicieron utilizando la paquetería STATA 10.0 (Stata Corp., College Station, Tex). Las variables continuas se expresan como medias y desviación estándar (DE) o medianas y amplitud, dependiendo de la distribución de los datos. Las variables categóricas se describen como proporciones. Para comparar las características demográficas y clínicas entre los tres países participantes se recurrió al análisis de varianza (ANOVA), así como a la prueba de chi cuadrado. Los resultados se presentan en forma combinada para los tres países.

\section{CONSIDERACIONES ÉTICAS}

El Comité de Ética institucional en cada país revisó en forma independiente el protocolo del proyecto para dar su aprobación, y todos los sujetos participantes dieron su consentimiento informado por escrito para participar en el estudio.

\section{RESULTADOS}

\section{RECLUTAMIENTO}

Entre los meses de noviembre y diciembre de 2011 se identificaron a un total de 243 participantes potenciales en los tres países, de ellos, 55 resultaron elegibles $y$, finalmente, fueron 45 sujetos con prehipertensión los que aceptaron participar y fueron incluidos (12 en Guatemala, 17 en Perú y 16 en Argentina) (Figura 3).

La edad promedio de los participantes fue de $45,5(8,8)$ años; $64,4 \%$ eran del sexo femenino, y 75,6\% contaban con siete o más años de escolaridad. El promedio de la presión arterial sistólica y diastólica fue $127,2(6,8) \mathrm{mm} \mathrm{Hg}$ y $78(7,3) \mathrm{mm} \mathrm{Hg}$, respectivamente. El promedio del índice de masa corporal y de la circunferencia fue $31,7(5,8)$ y 101 (11) $\mathrm{cm}$, respectivamente. No hubo diferencias significativas entre los valores recolectados en los tres países.

En cuanto al uso del teléfono celular: 32 participantes tenían un servicio de prepago; ocho habían cambiado su número durante el último año; tres mencionaron no usar su teléfono para hacer llamadas; cinco no sabían cómo abrir un mensaje de texto en su celular, y cinco mencionaron que no abrían los mensajes de texto si no reconocían el número celular del cual era originado.

\section{MEDIDAS DE RESULTADO}

Para la fase de seguimiento se logró contactar a 43 de los 45 participantes (95\%). De ellos, 38 (84\%) recibió

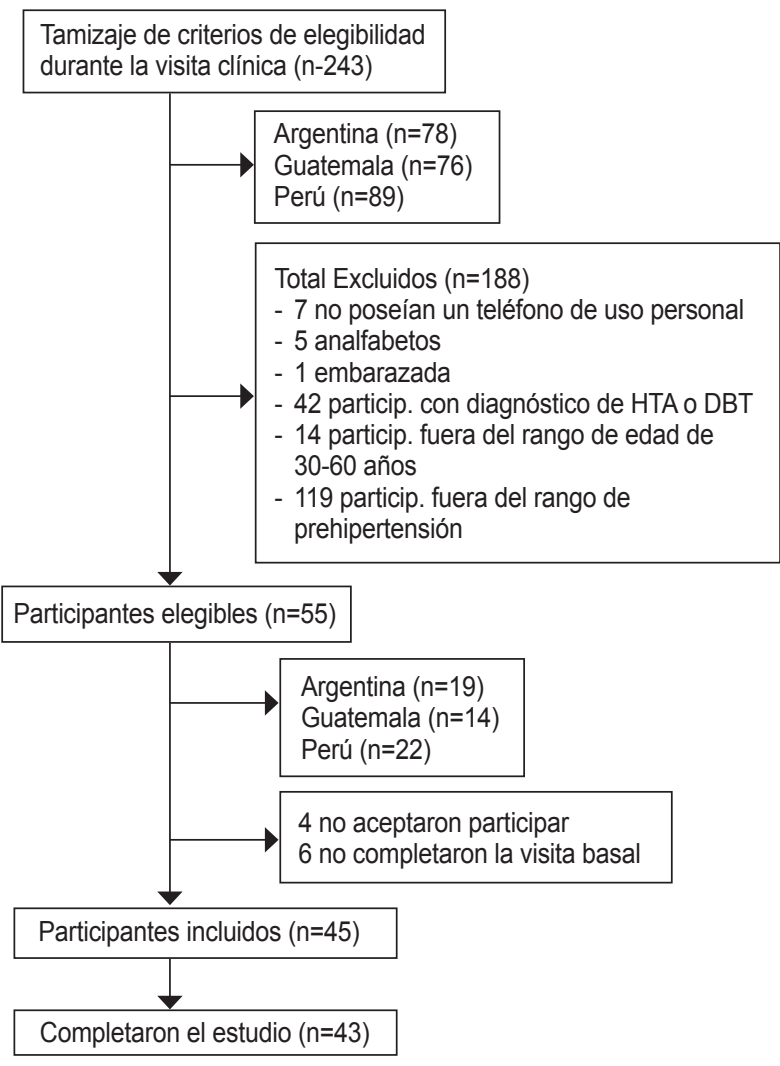

Figura 3. Diagrama de flujo para describir el tamizaje y reclutamiento de los participantes

el paquete completo de la intervención, que incluyó la llamada de introducción y la llamada de consejería, así como un mensaje de texto semanal. Dos de los participantes no pudieron ser contactados en su teléfono celular, y cinco fueron contactados, pero no se pudo completar la entrevista motivacional dentro del mes de estudio. Ningún participante abandonó el estudio. El número promedio de llamadas efectuadas a fin de poder contactar con el participante para la llamada de introducción fue de 2 con un rango de 1-9, y de 2,3 para la entrevista motivacional, con un rango de 1-8 intentos. La duración media de la llamada motivacional fue de 31 minutos, con un rango de 10-60 minutos.

\section{ACEPTABILIDAD A LA INTERVENCIÓN DE SALUD MÓVIL PROPUESTA}

Con base en 31 entrevistas semiestructuradas conducidas mediante llamada telefónica una vez concluido el mes de intervención, los participantes que completaron la intervención de salud móvil expresaron que las llamadas telefónicas y los mensajes de texto recibidos fueron útiles, y que les motivaron a modificar la conducta objetivo. 
Esto motiva a las personas a hacer las cosas diferentes. Yo cambié la forma en que como (Hombre, 38 años, Argentina).

Me gustaron las sugerencias y los consejos que me dio la nutricionista para reducir la cantidad de sal que como (Mujer, 32 años, Guatemala).

Me gustó que los mensajes de texto me den ideas y me ayuden a recordar que debo cuidar mi salud y mi corazón (Mujer, 58 años, Argentina).

Además, los participantes mencionaron que el tono de las llamadas fue muy amistoso, y que estaban receptivos hacia una intervención de salud móvil.

La nutricionista fue muy amistosa y atenta, y me explicó las cosas bien (Mujer, 39 años, Perú).

Sin embargo, dos participantes expresaron que aunque les pareció que la consejería a través de salud móvil era útil, ellos necesitaban del contacto cara-a-cara con un profesional de la salud para lograr un cambio en su estilo de vida.

En cuanto a los mensajes de texto, los participantes expresaron que estos fueron útiles, que les motivaron y les hicieron recordar los cambios que habían elegido hacer.

Hay algunas lecturas que resultan aburridas y a mí no me gusta eso, aunque sean textos. Yo definiría estos mensajes como divertidos y como lectura fácil, y que logran interesar e informar a uno. De acuerdo con el formato utilizado (Hombre, 32 años, Perú).

Me gusta que me den ideas y hacen que uno recuerde que debe consumir más frutas y verduras, que las puedo combinar con otros alimentos, como arroz con verduras (Mujer, 52 años, Perú).

Me gustan los mensajes que le motivan a uno, porque al final te dicen que lo puedes hacer (Hombre, 30 años, Guatemala).

\section{BARRERAS PARALAENTREGADELAINTERVENCIÓN}

La principal barrera reportada por las nutricionistas para entregar la intervención de salud móvil como había sido programada, fue la interrupción que ocurría durante las llamadas motivacionales como consecuencia de problemas en la recepción de la señal de telefonía celular. En forma particular, $17 \%$ de los participantes reportaron que la entrevista motivacional se vio interrumpida y tuvo que ser reagendada.
En una ocasión se cortó la señal, pero la nutricionista me volvió a llamar. Sin embargo, yo tenía mala recepción celular, y tuve que cambiar de sitio para poder escuchar bien (Mujer, 45 años, Perú).

Durante la llamada, yo no entendía algunas de las cosas que me decía la nutricionista, asi que tuve que cambiarme hacia otro lugar y pedirle a la nutricionista que repitiera lo que me había dicho (Hombre, 41 años, Argentina).

Las nutricionistas también reportaron dificultades para contactar a los participantes debido a otras actividades que competían con la llamada, por ejemplo, el trabajo.

A veces, una no tiene tiempo de tomar la llamada, porque me encontraba en camino hacia el trabajo, asi que es mejor recibir los mensajes de texto porque uno los puede guardar y leerlos más tarde (Mujer, 47 años, Argentina).

En cuanto a la entrega de los mensajes de texto, la información recolectada durante las llamadas semiestructuradas señaló que algunos participantes que recibieron la llamada motivacional no recibieron los dos mensajes de texto que fueron enviados después de la misma. Las razones ofrecidas para explicar esto fueron: mala señal de telefonía celular, buzón del celular lleno, y celulares compartidos, perdidos o robados.

Yo recibi el primer mensaje, pero no el segundo. Esto puede deberse a que a veces el celular no tiene señal (Hombre, 30 años, Guatemala).

Otros participantes no leyeron los mensajes de texto porque no sabían cómo abrirlos.

Sonaba como si fueran mensajes, pero no sé cómo leerlos (Mujer, 48 años, Perú).

\section{SATISFACCIÓN DEL USUARIO CON LA PLATAFORMA}

Las nutricionistas reportaron que la plataforma basada en Internet fue fácil de aprender y sencilla de usar. Comentaron que les ayudó a guiar la llamada de consejería con los participantes y reportaron un buen desempeño de la herramienta.

\section{DISCUSIÓN}

Los resultados obtenidos muestran que las llamadas de consejería y los mensajes de texto por telefonía celular para promover cambios en los estilos de vida fueron bien aceptados por los participantes. En este estudio 
piloto fue posible ponerse en contacto con la mayoría de los participantes, aunque en algunos casos resultó un reto contactarlos en su teléfono celular.

Se encontraron algunas barreras para llevar a cabo la llamada de consejería. La principal barrera fue la interrupción en la llamada debido a mala recepción de la señal de telefonía celular. Otra barrera que encontraron las nutricionistas fue la falta de capacidad de los participantes para atender debido a labores competitivas como el trabajo. Los participantes reportaron problemas con su señal de telefonía celular y con la recepción de los mensajes de texto. Posibles explicaciones para estos hallazgos son la pobre cobertura de telefonía celular en estas zonas de bajos recursos, y que la mayoría de los participantes tenía un servicio de prepago, por lo que los celulares se apagan cuando se les acaba el crédito.

En general, los participantes sintieron que la llamada de entrevista motivacional fue benéfica y útil para ayudarles a adoptar estilos de vida saludables. Además, los participantes mencionaron que los mensajes funcionaron como recordatorio y para motivar la acción en las conductas objetivo. El contenido, el tiempo y la frecuencia de las llamadas y de los mensajes de texto fue aceptable, y ningún participante expresó efectos indeseables de la intervención (por ejemplo, la percepción de que se invadía su privacidad, o eran intrusivas). Este fue un aspecto importante de considerar, puesto que no hay evidencia publicada en relación con la frecuencia de mensajes de texto utilizados en el cuidado preventivo o en la promoción de la salud (26). En este sentido, la evidencia en relación con el mejor momento de enviar mensajes de texto y su frecuencia varía entre diferentes estudios, así como en relación con el resultado buscado (dejar de fumar, pérdida de peso, aumento en la actividad física), desde una a cinco veces por día hasta una vez por mes ${ }^{(27-29)}$

En este estudio también se encontró que algunos participantes no sabían cómo abrir un mensaje de texto. En este sentido, es importante evaluar el conocimiento y familiaridad con la tecnología relacionada con la salud móvil, y educar a los participantes al implementar una estrategia de salud móvil, puesto que esto puede afectar tanto la forma en que se desarrolla la intervención como su adopción futura ${ }^{(30)}$. La implementación de estrategias de salud móvil requiere la habilidad combinada de las personas receptoras para manejar la tecnología y adquirir la información necesaria para tomar las decisiones adecuadas para lograr el cambio hacia estilos de vida saludables. La plataforma diseñada para ofrecer la intervención mostró ser amigable con el usuario, especialmente porque resultó útil a las nutricionistas para guiar la llamada motivacional e individualizar el grupo de mensajes de texto que se enviaron a cada participante.

Una limitación de este estudio fue su corta duración, explicable por su carácter piloto. Se espera que las intervenciones orientadas a modificar los estilos de vida duren hasta que los individuos en alto riesgo de desarrollar ECV logren el cambio y lo mantengan. La aceptabilidad y la adherencia a este tipo de intervención pueden cambiar cuando estas intervenciones se prolongan por largo tiempo. Una segunda limitación del estudio fue la inclusión de una mayor proporción de mujeres con prehipertensión que de hombres, ya que la prevalencia de esta condición es mayor entre el sexo masculino. Sin embargo, hay un menor uso de los servicios de salud primaria por parte de los hombres, y por lo tanto, la muestra estudiada representa a la población que acude a solicitar servicios de atención primaria en estos países en donde puede integrarse una intervención de salud móvil (31).

El uso de Internet y de intervenciones basadas en salud móvil constituye un medio prometedor para ofrecer intervenciones diseñadas para la promoción de la adopción de estilos de vida saludables. Sin embargo, la penetración de Internet, en comparación con el uso de la telefonía celular, es baja en zonas de bajos recursos en países en vías de desarrollo ${ }^{(32)}$. De acuerdo con otros estudios que han investigado la combinación de tecnologías para promover conductas saludables, nuestros resultados sugieren que el uso de la salud móvil fue bien aceptado y puede ampliar la extensión de la atención preventiva cuando se integra a los servicios de salud ${ }^{(33-35)}$. El uso de teléfonos celulares y mensajes de texto ya son parte de la vida diaria, aun en países de bajos y medianos ingresos. Su uso está aumentando,

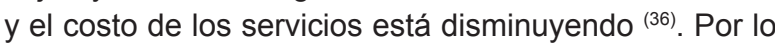
tanto, impresiona que la salud móvil sea potencialmente una herramienta fuerte para mejorar la salud, especialmente tratándose de poblaciones vulnerables, debido a su bajo costo, amplitud de uso, simplicidad y aplicabilidad en una amplia variedad de conductas y condiciones relacionadas con la salud. Sin embargo, aún se requiere de la disponibilidad de una buena infraestructura móvil en países de bajos y medianos ingresos para poder ofrecer servicios de salud móvil en zonas de bajos recursos ${ }^{(37-39)}$.

Las estrategias basadas en la telefonía celular pueden facilitar la consulta con flexibilidad en relación con tiempo y lugar, y por lo tanto, reducir los costos y ampliar la cobertura y la accesibilidad de las intervenciones orientadas a la promoción de la salud en zonas de bajos recursos en países en vías de desarrollo. En este sentido, la salud móvil constituye una oportunidad para 
mejorar la salud de las personas y como un medio para enfrentar las limitaciones que enfrentan los sistemas de salud en Latinoamérica, relacionadas con la falta de recursos, una alta carga de enfermedad a expensas de un incremento de las enfermedades crónicas, la pobreza y el crecimiento poblacional.

Contribuciones de autoría: $A B$ y $A R$ han participado en la concepción del artículo, su redacción y aprobación de la versión final. FDC, RK y PL han participado en la recolección de los datos y en la revisión del artículo. AF ha realizado la asesoría técnica del estudio. $A B$ realizó el análisis e interpretación de datos. Además MRZ, JJM, HM y AR obtuvieron el financiamiento para el estudio, lo han revisado críticamente y aprobaron la versión final.

Fuentes de financiamiento: este proyecto fue financiado por los Institutos Nacionales de Salud de los Estados Unidos de América, a través del National Heart, Lung, and Blood Institute (HHSN268200900028C).

Conflictos de interés: los autores declaran no tener conflictos de interés.

\section{REFERENCIAS BIBLIOGRÁFICAS}

1. Gaziano TA. Reducing the growing burden of cardiovascular disease in the developing world. Health Aff (Millwood). 2007 Jan-Feb;26(1):13-24.

2. Tibazarwa KB, Damasceno AA. Hypertension in developing countries. Can J Cardiol. 2014 May;30(5):52733. doi: 10.1016/j.cjca.2014.02.020.

3. Huang Y, Wang S, Cai X, Mai W, Hu $\mathrm{Y}$, Tang $\mathrm{H}$, et al. Prehypertension and incidence of cardiovascular disease: a meta-analysis. BMC Med. 2013 Aug 2;11:177. doi: 10.1186/1741-7015-11177.

4. Rubinstein AL, Irazola VE, Poggio R, Bazzano L, Calandrelli M, Lanas Zanetti FT, et al. Detection and followup of cardiovascular disease and risk factors in the Southern Cone of Latin America: the CESCAS I study. BMJ Open. 2011 May 26;1(1):e000126. doi: 10.1136/bmjopen-2011-000126.

5. Pan American Health Organization. The Central America Diabetes Initiative (CAMDI): Survey of Diabetes, Hypertension and Chronic Disease Risk Factors. Washington, D.C: PAHO; 2011.

6. Miranda JJ, Bernabe-Ortiz A, Smeeth L, Gilman RH, Checkley W , CRONICAS Cohort Study Group. Addressing geographical variation in the progression of non-communicable diseases in Peru: the CRONICAS cohort study protocol. BMJ Open. 2012 Jan 11;2(1):e000610. doi: 10.1136/bmjopen-2011-000610.

7. Chobanian AV, Bakris GL, Black HR, Cushman WC, Green LA, Izzo JL, Jr., et al. The Seventh Report of the Joint National Committee on Prevention, Detection, Evaluation, and Treatment of High Blood Pressure: the JNC 7 report. JAMA. 2003 May

\section{1;289(19):2560-71.}

8. Ebrahim S, Smith GD. Systematic review of randomised controlled trials of multiple risk factor interventions for preventing coronary heart disease. BMJ. 1997 Jun 7;314(7095):1666-74.

9. James PA, Oparil S, Carter BL, Cushman WC, Dennison-Himmelfarb C, Handler J, et al. 2014 evidencebased guideline for the management of high blood pressure in adults: report from the panel members appointed to the Eighth Joint National Committee (JNC 8). JAMA. 2014 Feb 5;311(5):507-20. doi: 10.1001/ jama.2013.284427.

10. Kohl LF, Crutzen R, de Vries NK. Online prevention aimed at lifestyle behaviors: a systematic review of reviews. J Med Internet Res. 2013 Jul 16;15(7):e146. doi: 10.2196/ jmir.2665.

11. Webb TL, Joseph J, Yardley L, Michie S. Using the internet to promote health behavior change: a systematic review and meta-analysis of the impact of theoretical basis, use of behavior change techniques, and mode of delivery on efficacy. J Med Internet Res. 2010 Feb 17;12(1):e4. doi: 10.2196/jmir.1376.

12. Istepanian $R$, Laxminarayan $S$, Pattichis CS. M-Health: Emerging Mobile Health Systems. Topics in Biomedical Engineering. London: Springer; 2006.

13. Germanakos P, Mourlas C, Samaras G. A Mobile Agent Approach for Ubiquitous and Personalized eHealth Information Systems. Proceedings of the Workshop on 'Personalization for e-Health' of the 10th International Conference on User Modeling (UM'05); July 29th; Edinburgh: 2005.

14. Mechael P, Batavia H, Kaonga N, Searle $S$, Kwan A, Goldberger A, et al. Barriers and gaps affecting mHealth in low and middle income countries: Policy white paper. New York NY: The Earth Institute, Columbia University; 2010.

15. Krishna S, Boren $S$ A, Balas AE. Healthcare via cell phones: a systematic review. Telemed J E Health. 2009 Apr;15(3):231-40. doi: 10.1089/ tmj.2008.0099.

16. Cole-Lewis H , Kershaw T. Text messaging as a tool for behavior change in disease prevention and management. Epidemiol Rev. 2010;32:56-69. doi: 10.1093/epirev/mxq004.

17. Fjeldsoe BS, Marshall AL, Miller YD. Behavior change interventions delivered by mobile telephone shortmessage service. Am J Prev Med. 2009 Feb;36(2):165-73. doi: 10.1016/j. amepre.2008.09.040.

18. Beratarrechea A, Lee AG, Willner JM, Jahangir E, Ciapponi A, Rubinstein A. The impact of mobile health interventions on chronic disease outcomes in developing countries: a systematic review. Telemed J E Health. 2014 Jan;20(1):75-82. doi: 10.1089/ tmj.2012.0328.

19. Krishna S, Balas EA, Boren SA, Maglaveras N. Patient acceptance of educational voice messages: a review of controlled clinical studies. Methods Archive. 2002;41(5):360-9.

20. Miller WR, Rose GS. Toward a theory of motivational interviewing. Am Psychol. 2009 Sep;64(6):527-37. doi: 10.1037/a0016830.

21. Di Noia J, Prochaska JO. Mediating variables in a transtheoretical model dietary intervention program. Health Educ Behav. 2010 Oct;37(5):753-62. doi: $10.1177 / 1090198109334897$.

22. Prochaska JO, DiClemente CC. Stages and processes of self-change of 
smoking: toward an integrative model of change. J Consult Clin Psychol. 1983 Jun;51(3):390-5.

23. Diez-Canseco F, Zavala-Loayza JA, Beratarrechea A, Kanter R, RamirezZea M, Rubinstein A, et al. Design and Multi-Country Validation of Text Messages for an mHealth Intervention for Primary Prevention of Progression to Hypertension in Latin America. JMIR mHealth and uHealth. 2015 Feb 18;3(1):e19. doi: 10.2196/ mhealth.3874.

24. Baron S, Goutard F, Nguon K, Tarantola A. Use of a text messagebased pharmacovigilance tool in Cambodia: pilot study. J Med Internet Res. 2013 Apr 16;15(4):e68. doi: 10.2196/jmir.2477.

25. FrontlineSMS Cloud.com [Internet]. Washington, DC: the Frontline team. [citado el 25 de Enero de 2015]. Disponible en: http://www. frontlinesms.com/.

26. Vodopivec-Jamsek V, de Jongh T, Gurol-Urganci I, Atun R, Car J. Mobile phone messaging for preventive health care. Cochrane Database Syst Rev. 2012 Dec 12;12:CD007457. doi: 10.1002/14651858.CD007457.pub2.

27. Shaw R, Bosworth H. Short message service (SMS) text messaging as an intervention medium for weight loss: A literature review. Health Informatics J. 2012 Dec;18(4):235-50. doi: $10.1177 / 1460458212442422$.

28. Buchholz SW, Wilbur J, Ingram D, Fogg L. Physical activity text messaging interventions in adults: a systematic review. Worldviews Evid-Based Nurs. 2013 Aug;10(3):163-73. doi: 10.1111/ wvn.12002.

29. Balmford J, Borland R. How do smokers use a smoking cessation text messaging intervention?. Nicotine Tob Res. 2014 Dec;16(12):1586-92. doi: $10.1093 /$ ntr/ntu 111 .

30. Norman C. eHealth literacy 2.0: problems and opportunities with an evolving concept. J Med Internet Res. 2011 Dec 23;13(4):e125. doi: 10.2196/jmir.2035.

31. Douglas FC, Greener J, van Teijlingen E, Ludbrook A. Services just for men? Insights from a national study of the Well Men Services Pilots. BMC Public Health. 2013 May 1;13:425. doi: 10.1186/1471-2458-13-425.

32. Wallsten S. Regulation and Internet Use in Developing Countries [Internet]. Washington, DC: World Bank Policy Research; 2002 [citado el 22 de enero de 2015]. Disponible en: http://papers. ssrn.com/sol3/papers.cfm?abstract_ $\mathrm{id}=366100 \&$ download $=$ yes

33. Korhonen I, Mattila E, Ahtinen A, Salminen J, Hopsu L, Lappalainen R, et al. Personal health promotion through personalized health technologies Nuadu experience. Conf Proc IEEE Eng Med Biol Soc. 2009;2009:316-9. doi: 10.1109/IEMBS.2009.5333550.

34. Mattila E, Parkka J, Hermersdorf M, Kaasinen J, Vainio J, Samposalo K, et al. Mobile diary for wellness management-results on usage and usability in two user studies. IEEE Trans Inf Technol Biomed. 2008 Jul;12(4):501-12. doi: 10.1109/TITB.2007.908237.

35. Mattila E, Lappalainen R, Parkka J, Salminen J, Korhonen I. Use of a mobile phone diary for observing weight management and related behaviours. J Telemed Telecare. 2010;16(5):260-4. doi: 10.1258/jtt.2009.091103.

36. Mechael P. The Case for mHealth in Developing Countries. Innovations: Technology, Governance, Globalization. 2009;4(1):103-18.

37. Rice RE , Katz JE. Comparing internet and mobile phone usage :digital divides of usage, adoption, and dropouts. Telecomm Policy. 2003;27(8-9):597-623.

38. Lim MS, Hocking JS, Hellard ME, Aitken CK. SMS STI: a review of the uses of mobile phone text messaging in sexual health. Int J STD AIDS. 2008 May;19(5):287-90. doi: 10.1258/ ijsa.2007.007264.

39. Franklin VL, Waller A, Pagliari C, Greene SA. A randomized controlled trial of Sweet Talk, a text-messaging system to support young people with diabetes. Diabet Med. 2006 Dec;23(12):1332-8.

Correspondencia: Andrea Beratarrechea

Dirección: Emilio Ravignani 2024 (C1414CPV)

Teléfono: (+54-11) 4777-8767

Correo electrónico: aberatarrechea@iecs.org.ar

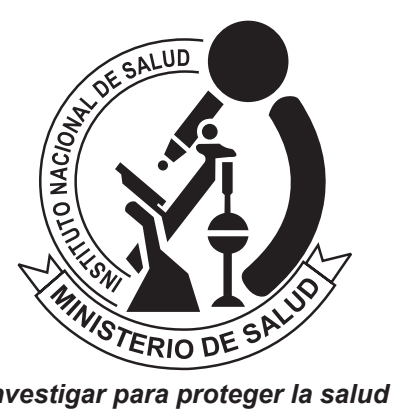

REVISTA PERUANA DE MEDICINA EXPERIMENTAL Y SALUD PÚBLICA CUMPLIENDO SUS METAS Y PROYECTÁNDOSE AL FUTURO

\section{Visite los contenidos de la revista en: www.ins.gob.pe/rpmesp}

